Zhao, J., Seppänen, O., and Peltokorpi, A. 2020. "Applying Heat Maps to Define Workspace in Construction Based on Real-Time Tracking System with Coordinate Positioning Information." In: Tommelein, I.D. and Daniel, E. (eds.). Proc. $28^{\text {th }}$ Annual Conference of the International Group for Lean Construction (IGLC28), Berkeley, California, USA, doi.org/10.24928/2020/0014, online at iglc.net.

\title{
APPLYING HEAT MAPS TO DEFINE WORKSPACE IN CONSTRUCTION BASED ON REAL-TIME TRACKING SYSTEM WITH COORDINATE POSITIONING INFORMATION
}

\author{
Jianyu Zhao ${ }^{1}$, Olli Seppänen², and Antti Peltokorpi ${ }^{3}$
}

\begin{abstract}
The construction jobsites are regarded as highly dynamic and chaotic environment for both workers and management. To ensure efficient task performances, workspace is a key resource in construction sites. Nowadays the technology has enabled better management of onsite resources through location-based methods within lean construction principles, such as Location-Based Management System (LBMS) and Takt planning. Those methods aim to optimize workspace in construction sites consisting of multiple tasks and to eliminate waste related to product and labour flows. To contribute to enhancing those lean construction approaches with attempts to obtain real and objective data, we have developed a real-time positioning system based on Bluetooth Low Energy (BLE) technology where coordinates of workers can be obtained, and heat maps of task-related workspace are generated in real time. This paper aims to develop automated process of generation of workspace based on coordinates of workers and to explore various uses of workspace heat maps to serve the purposes of lean construction principles. The successful implementation of the system in China is analysed and the results suggest that heat maps which use dimensional and temporal positioning data, could be an efficient and convenient method to define workspace of crews onsite.
\end{abstract}

\section{KEYWORDS}

Lean construction, real-time indoor positioning, heat-maps, intelligent construction site, onsite management.

\section{INTRODUCTION}

Construction sites are often in chaotic environment which leads to massive efficiency loss and waste due to managerial reason and resource mishandling (Akhavian et al. 2016). Over the last past years, previous studies have confirmed that there is substantial room for productivity improvement in construction industry in terms of scheduling, labor

1 Doctoral Candidate, Department of Civil Engineering, Aalto University, Espoo Finland, jianyu.zhao@aalto.fi, orcid.org/0000-0001-8519-617.

2 Associate Professor, Department of Civil Engineering, Aalto University, Espoo Finland, olli.seppanen@aalto.fi, orcid.org/0000-0002-2008-5924

3 Assistant Professor, Department of Civil Engineering, Aalto University, Espoo Finland, antti.peltokorpi@aalto.fi, orcid.org/0000-0002-7939-6612 
training and quality control (Arditi and Mochtar 2000). Therefore, there is a need for automatic-generated information for onsite resource management such as labors, materials and equipment (Zhao et al. 2018).

Workspace is a key resource in construction sites that are required by construction activities (Akinci et al. 2002). Alongside workspace management in construction, several lean methods such as Location-Based Management System (LBMS) and Takt Time Planning (TTP) are also applied to eliminate waste and enhance productivity at work locations onsite. For instance, LBMS requires the Location Breakdown Structure (LBS) (Frandson et al. 2015) where each task is at a hierarchy and includes one or several work locations (Seppänen et al. 2010). TTP is aiming to decrease the variability of different tasks by imposing standard production rate throughout "right sized geographic areas" onsite (Takt area) (Emdanat et al. 2016). Therefore, workspace in construction plays important roles for both space management in construction and lean approaches to be applied, such as LBMS and TTP with goals for continuous flows of work through production areas during different work phases (Frandson et al. 2015). Workspace generation by activities onsite requires spatiotemporal data necessary to represent in four dimensions but it is difficult for project managers to obtain spatiotemporal data manually (Akinci et al. 2002) due to large efforts of data collection. There are attempts to automate generation of workspace such as Akinci et al. to reduce the amount of data entered by the user but their research remains in planning phase and those space requirements with spatial information have not been compared with real situations of workers conducting tasks onsite.

In previous studies, researchers have tried to apply three-dimensional modeling to detect and track moving or static object boundaries in real-time based on video cameras (Teizer et al. 2007). Later on heat map generation has been proposed by Golovina et al (2016), where they applied heat map generation in construction to map at-risk behaviors of workers, but yet the application has been served for safety planning instead of operations management purposes. Badihi et al (2019) have implemented a Bluetooth Low Energy (BLE) tracking system according to trilateration method to acquire the real-time location of resources and movement trajectory in jobsite. In their results, the formation from multiple single point of detected coordinates is subject to high deviation from the original paths, and they only carried out data analyses on a single-person simulation path for validating system's accuracy, but not yet on real worker's movement coordinates. Furthermore, they focused on technical requirement and description of system architecture but not on use cases from lean perspectives in construction.

This paper aims to develop automated process of workspace detection based on coordinates of workers and explore various uses of workspace heat maps to serve the purposes of lean construction principles. We applied the same tracking system as Badihi et al. (2019) but extend the method to generate the heat maps of workers' onsite presence, aiming to outline their workspace based on density of detected points. We believe through this research, the construction industry can benefit from automated workspace detection based on heat map generation from real-time tracking system, which complements other lean approaches in construction with goals to enhance management efficiency and eliminate the waste. The research questions of this paper are:

- How can a coordinate-based real-time tracking system be applied to real-size construction projects?

- How can heat maps of workers' presence onsite be used to serve lean approaches in construction such as takt time planning and uninterrupted presence detection? 


\section{METHODS}

\section{Case Selection}

A single-case study approach was chosen as a research method. The location of the case was in a Chinese city close to the capital Beijing. The construction project was undergoing a large amount of MEP work (mechanical, electrical and plumbing work) at the time of testing in a large shopping mall building. The real-time tracking system was implemented on the second floor at the north corner of the building where the MEP workers were scheduled to conduct pipeline-assembling task at the location (figure 1).

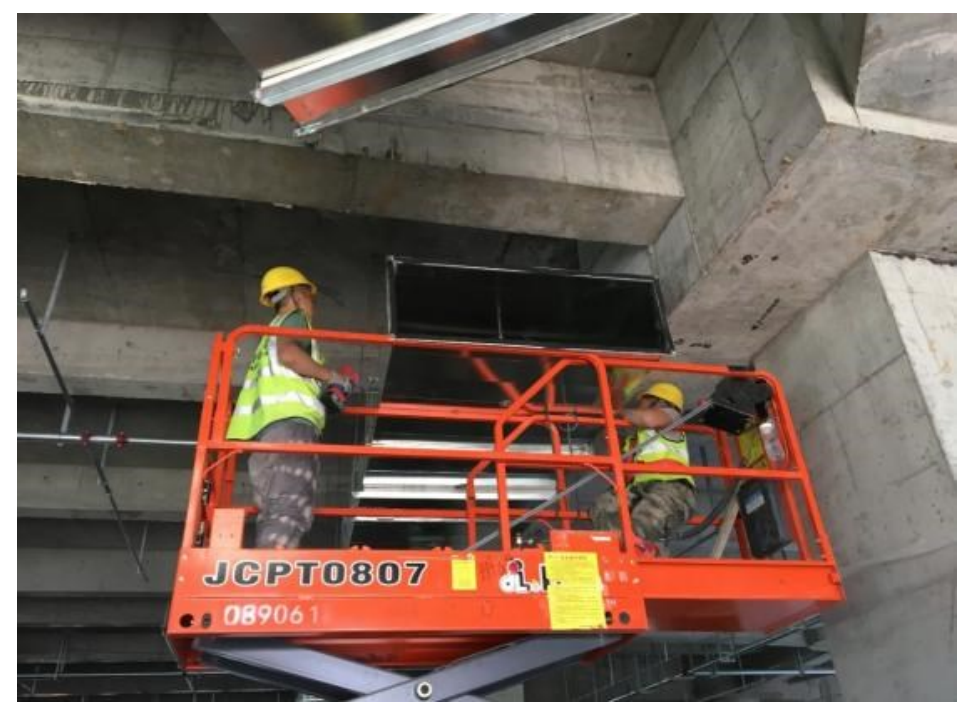

Figure 1: Pipeline-assembling task

The second floor is a big indoor open workspace (figure 2) with a regular grid of loadbearing pillars (nine meters away from each other), and the task of pipeline-assembling should proceed following the regular direction of continuous work (from west to east). Therefore, the layout of the test place and the task nature of the work is suitable for indoor positioning with the feature of coordinate detection and generation of heat maps.

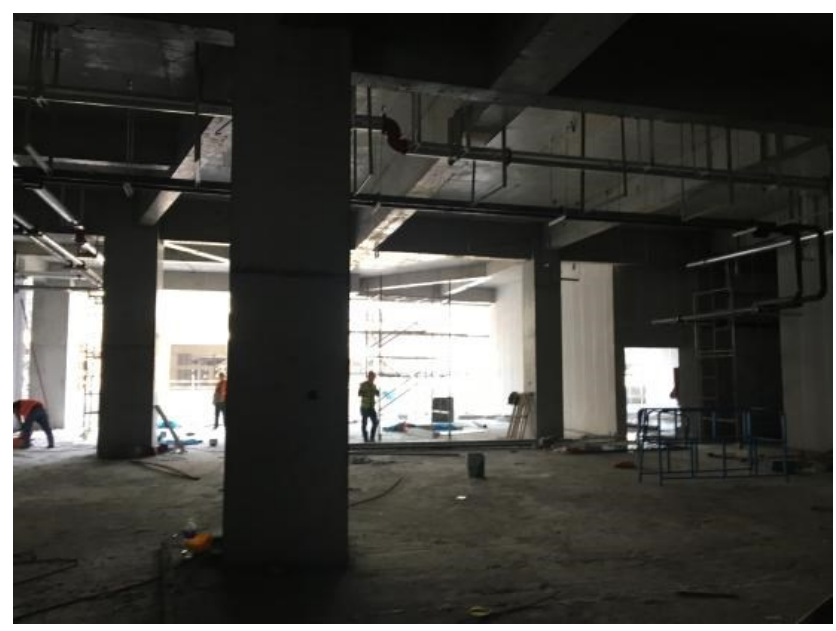

Figure 2: Indoor open working area in the jobsite 


\section{TRACKING SYSTEM INTEGRATED WITH COORDINATES}

Badihi et al (2019) have demonstrated an end-to-end BLE-based positioning system featured with coordinates shown in the figure 3 . This system consists of two independent data planes, namely, tracking data and management data. The management data plane is only used for managerial and administrative tasks and does not include positioning information. The system which is capable of positions and tracking individuals, material and tools with coordinates comprises of the following separated parts: (1) BLE beacons, which transmit data using Bluetooth Low Energy radio technology and can be detected by any Bluetooth compatible devices. These beacons with their unique MAC (Media Access Control) addresses are attached to the tracking objects. (2) Gateways, which are intermediate entities that collect data from beacons, aggregate and finally send them to the cloud. The data is transmitted in one second time interval. (3) Cloud, which is the brain of the tracking system and comprises of different modules. The cloud receives all the data using MQTT (Message Queuing Telemetry Transport) protocol. MQTT is a publish-subscriber based messaging protocol and works according to client-server model. The server or broker is located on the cloud and distributes all of the receiving data to the Analyzer module. Analyzer module is responsible for analyzing incoming raw data, removing the redundant data, gateway selection for the beacons and saving data in the database. In addition, the Analyzer module feeds the Positioning module that determines the coordination of the beacons relative to the reference gateway.

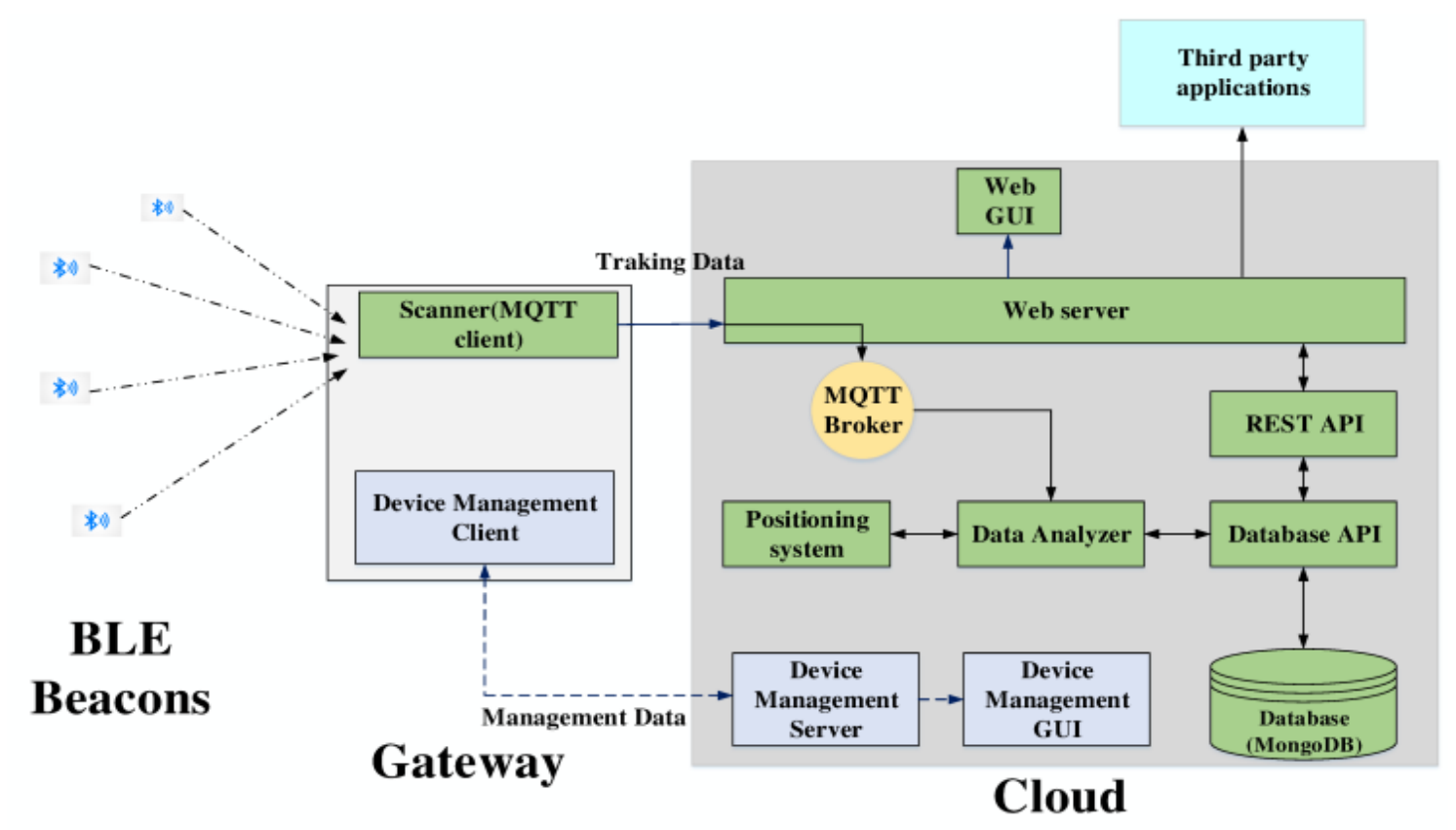

Figure 3: Overview of the tracking system (adopted from Badhi et al. 2019)

We followed the same infrastructure of the technology on the case where we aimed to obtain coordinate data of the workers onsite based on the illustrated system infrastructure and analyze its accuracy based on validation. We managed to implement seven gateways onsite and attach them to the building pillars approximately 1.5 meters above the floor. Each adjacent pillar is nine meters away from each other therefore an ideal coordinateintegrated floorplan can be modelled. We pinpointed each pillar with coordinate and set point $\mathrm{T} 2$ in figure 4 as our origin point. 


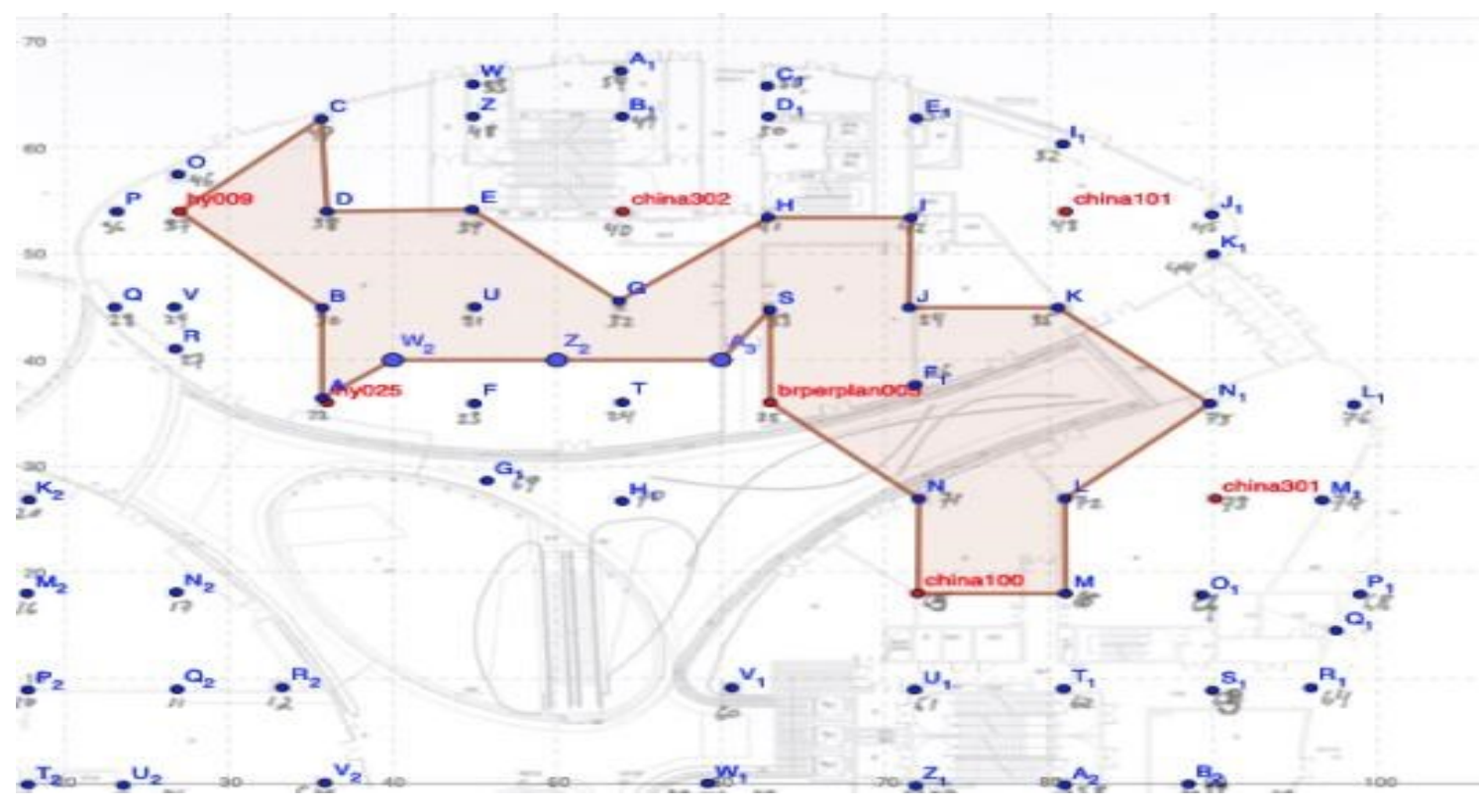

Figure 4: Floorplan with points in coordinates

\section{VALIDATION}

The red dots in figure 4 were the validation routine where a researcher simulated a worker's possible onsite path and compared the coordinates of each test point along the path with the system-captured records. Badhi et al. (2019) have carried out validation results based on three different measurement intervals on each test point along the path (30 second, 1 minute and 2 minutes). The measurement intervals refer to the time the researcher purposely has stayed at each test point before moving to the next point.

Table 1: Results of the validation (Table 1 in Badhi et al. 2019)

$\begin{array}{ccccc}\begin{array}{c}\text { Validation } \\ \text { measurement } \\ \text { intervals }\end{array} & \begin{array}{c}\text { X-coordinate } \\ \text { correlation }\end{array} & \begin{array}{c}\text { Y-coordinate } \\ \text { correlation }\end{array} & \begin{array}{c}\text { Average } \\ \text { deviation of } \\ \text { all recorded } \\ \text { points }(\mathrm{m})\end{array} & \begin{array}{c}\text { Standard } \\ \text { deviation of } \\ \text { all recorded } \\ \text { points }(\mathrm{m})\end{array} \\ \mathbf{2} \mathbf{~} \mathbf{~ i n} & 0.86 & 0.81 & 10.69 & 6.72 \\ \mathbf{1} \mathbf{~} \mathbf{m i n} & 0.67 & 0.71 & 14.06 & 9.71 \\ \mathbf{3 0} \mathbf{~ s e c} & 0.74 & 0.35 & 19.18 & 11.62\end{array}$

The results in table 1 suggest that with bigger measurement intervals, the average deviation and standard deviation of all recorded points are lower. The reason is that if a person stays at a test point longer (like 2 minutes), the more stability there is for the tracking system to detect the location of the person, thus more accurate of its coordinates. However, the lowest average deviation between actual test points and system-captured coordinates is more than 10 meters away and this is more than one pillar away in real situation. 


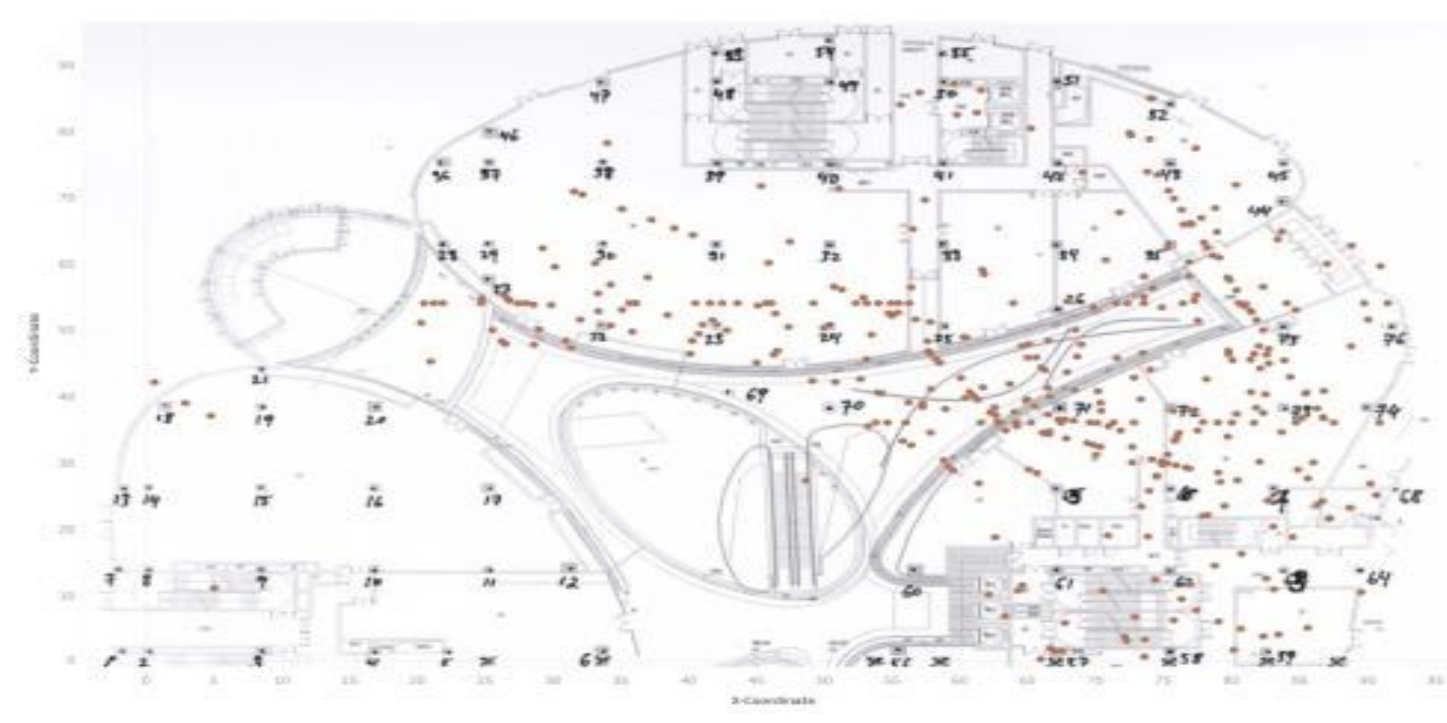

Figure 5: System-captured points for the 2-minute measurement interval

Figure 5 implies that for a certain set routine onsite, the system could display the points everywhere and they can hardly form a routine line as in figure 4 . Therefore, for a single captured point with coordinate, the system is unable to provide very accurate point indicated in the floorplan. This motivates the need of generation of heat maps based on density of captured points because heat maps can indicate overall fuzzy areas rather than single points according to the density of existence points onsite.

Based on the density of the points indicated in figure 5, we have therefore conducted the heat map of the validation movement routine in figure 6 . The light-colored area outlined the coverage of all detected points in figure 5 while the highlighted area outlined the researcher's actual movement line corresponded to routine in figure 4, except the small part missing from the upper area. The reason for this is that the movement reduction process from the system involves generating a great number of discrete points (coordinates) alongside the movement path, which impacts the deviation of single points in validation. However, in fact multiple points are generated per second to pinpoint the coordinate of the actual point in the system. In practice, there are more generated points as they are closer to the actual point, which makes higher density of the points in the figure. Therefore, it is reasonable to assume that deeper-colored area (higher density) is of greater probability to reflect the real place of a worker at a given time. This is also why we have seen in figure 6 that the detected points (lighter-colored areas) are all over places (with high deviation) but movement line (deeper-colored area) can nevertheless be displayed by the heatmaps based on density of system detected points. This implies that even though the proposed real-time tracking system may lack of accuracy in detail of single detected point with right coordinate, the heat maps based on density of movement point can still outline general areas of movement in real situation. Therefore, it provides evidence that heat maps can be an efficient approach to define overall workspace during any given time of the day. However, a plausible approach to decrease the overall variance is to place more gateways to improve the stability of the trilateration system, such as each gateway at one pillar onsite. 


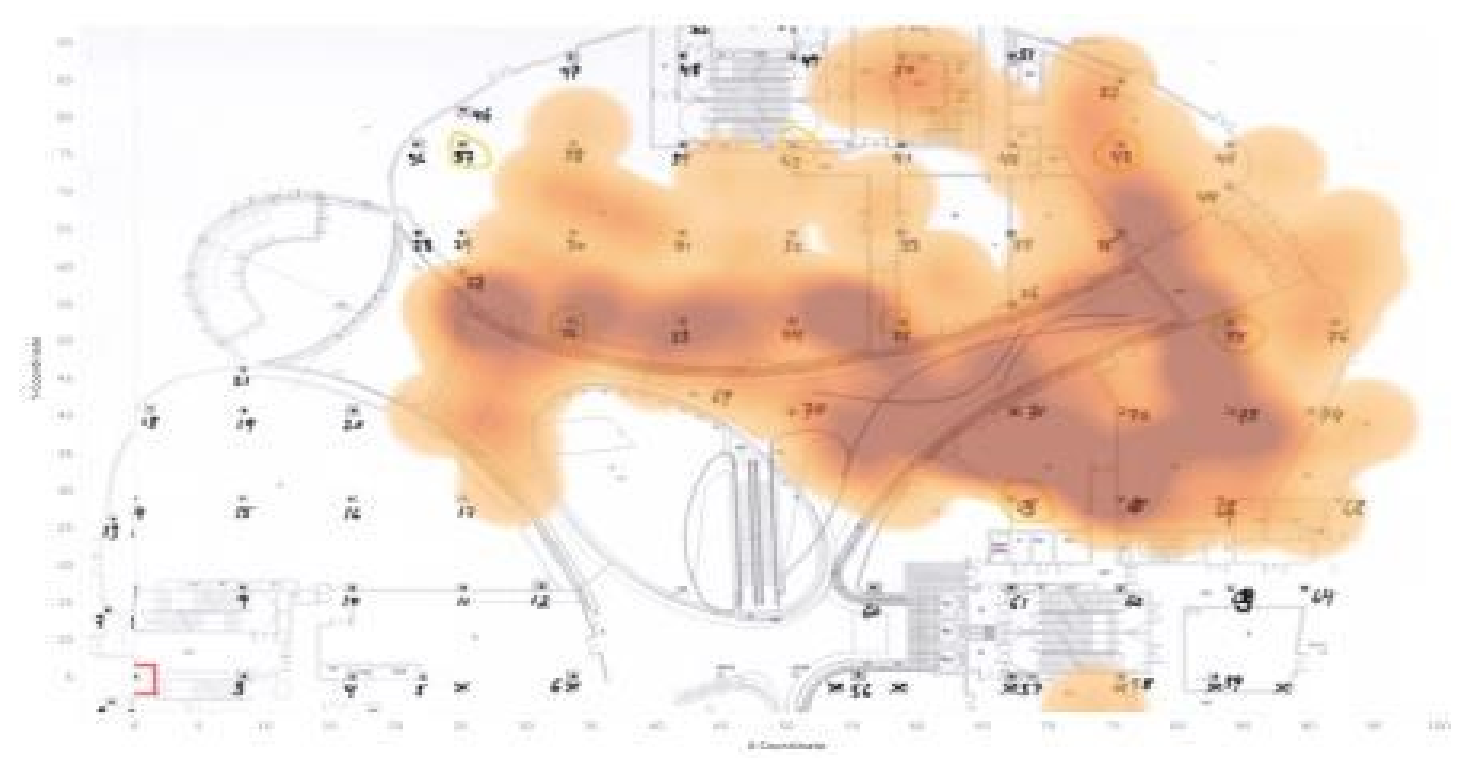

Figure 6: Heat map of the validation movement routine

\section{RESULTS}

\section{HEAT MAPS WITH HOURLY INTERVALS}

The heat map with hourly intervals can easily identify which hour of the day could be the workers' most likely-to-work time based on their movement density onsite. For instance, figure 7 is a heat-map change figure on May 30, 2019 onsite for MEP worker 16. The figure gives the following indications:

- The worker was detected for intensive onsite presence between 9am to $11 \mathrm{am}$ at the east corner of the test site, and between $2 \mathrm{pm}$ to $4 \mathrm{pm}$ at the middle and east corner of the test site, while he was out of site between $12 \mathrm{pm}$ to $1 \mathrm{pm}$.

- The workspace (the highlighted area) that the system has detected between $2 \mathrm{pm}$ to $4 \mathrm{pm}$ is much larger than the time between $9 \mathrm{am}$ to $11 \mathrm{am}$. More specifically, between $2 \mathrm{pm}$ to $3 \mathrm{pm}$ the workspace seems to match with the pipeline-assembly direction as a straight line, followed with the next hour movement where the workers may have conducted some logistic tasks everywhere around that detected working area.

- The heat map change figure throughout a day could quickly automate the movement density formation with dimensional and temporal parameters therefore fast check workers' workspace in different hours. 


\section{0_NEP worker 16}

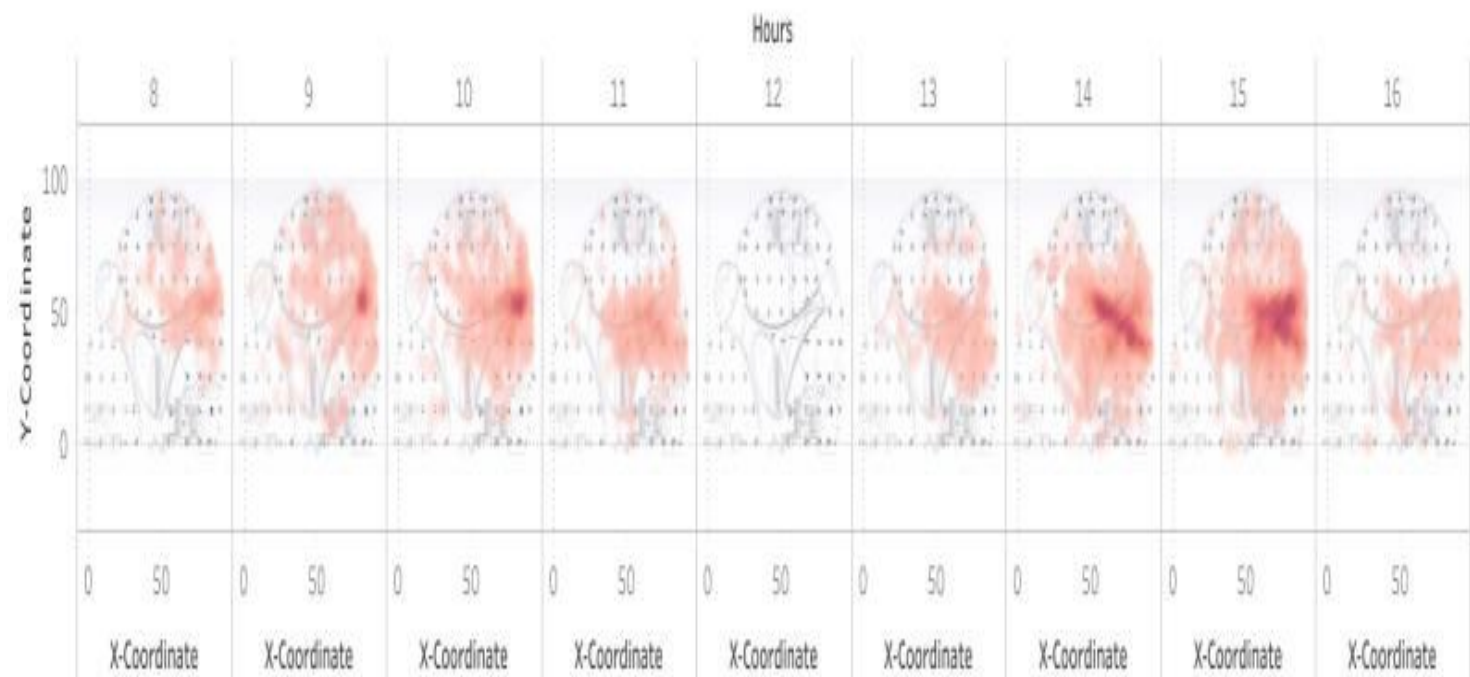

Figure 7: Hourly heat-map changes over time for MEP worker No. 16

\section{HEAT MAP IN DETAIL}

Heat maps can also be conducted in detail to interpret what has happened onsite based on the workers' coordinate information. Figure 8 demonstrates the captured workspace where one MEP worker has conducted his task between $2 \mathrm{pm}$ to $4 \mathrm{pm}$ with high level of uninterrupted presence detected during this time. The straight line during $2 \mathrm{pm}$ to $3 \mathrm{pm}$ time interval outlines a fuzzy coverage of the pipeline conducting area, which is correlated to this worker's onsite uninterrupted presence. It is reasonable to think that with deeper color of the heat map, the worker is more likely to conduct value-adding activities because higher frequency of position points is detected within the deepercolored area. Following this principle, the workspace detected between $3 \mathrm{pm}$ to $4 \mathrm{pm}$ for MEP worker 16 is enlarged (compared to workspace detected between $2 \mathrm{pm}$ and $3 \mathrm{pm}$ ) to a rectangular area bordered with point $24,70,73$ and 75 . This also suggests that the enlarged workspace during that hour was the required working space for the MEP worker in practice.

Therefore, heat maps connect dimensional and temporal data and integrate them into real-time tracking system where site managers are able to monitor site occurrences through an automated process. 


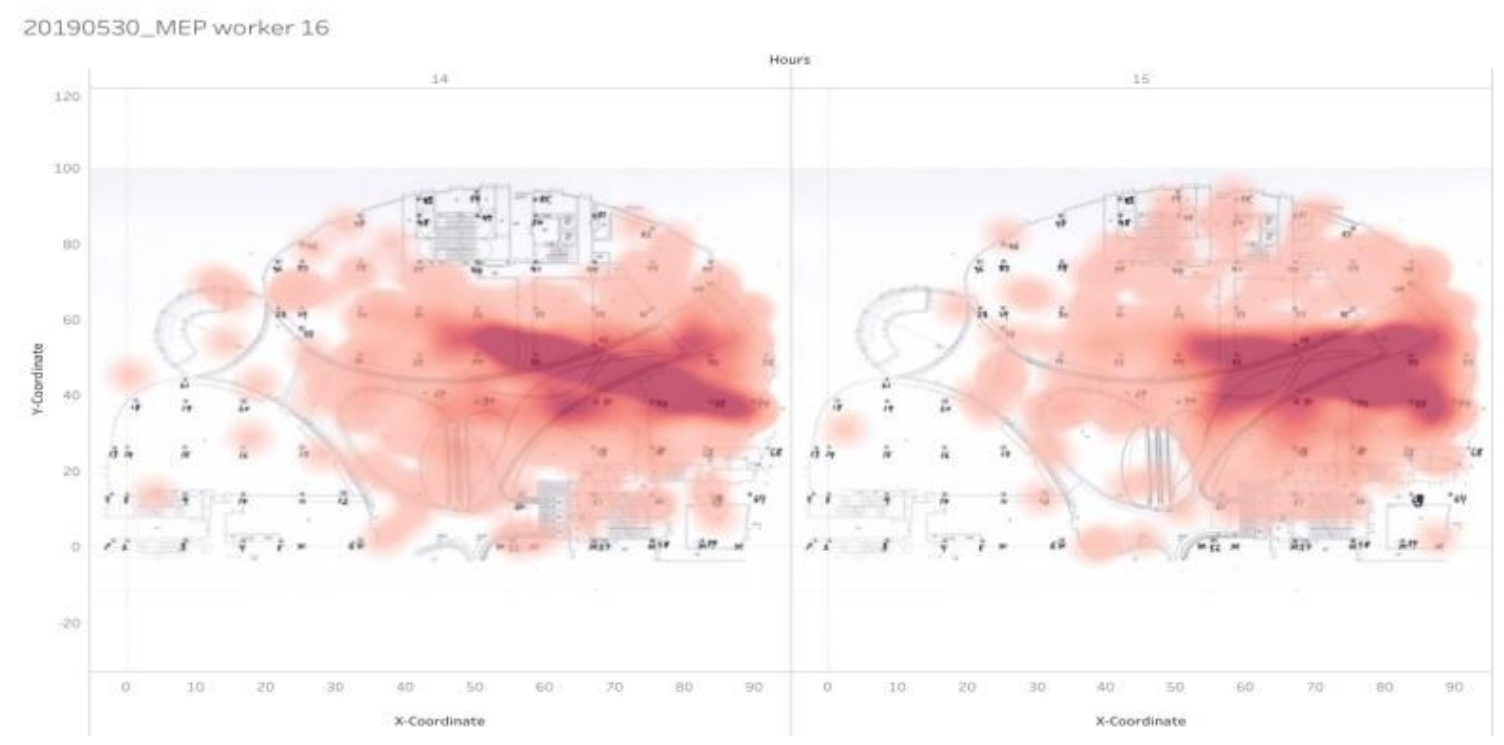

Figure 8: Heat maps in detail between 2pm to 4pm of MEP worker No. 16

\section{USE CASES OF HEAT MAP APPLICATION}

Based on our data in this case, we have explored several use cases of heat map applications:

- Analyzing takt areas. One key objective of lean thinking is to remove variability (Thomas et al. 2002). The concept of Takt time planning (TTP) is original from manufacture industry where production rates are set to reach the demand rate (e.g. Hopp and Spearman, 2008). Frandson et al (2015) have proposed that an important characteristic of TTP is for a set amount of time (Takt time), each trade is committed to finish their tasks in each assigned area (Takt area). Therefore, variability is decreased by using the assigned small area within the consistent duration (Takt time) and buffers are applied in each Takt area to prevent existing variability. For a project that implements Takt time planning, heat maps can automatically detect the workspace over time and compare them with assigned Takt area, to see if they match in real practice. Furthermore, heatmaps can also be generated to visualize how buffer capacity between tasks is followed and provide in-time feedbacks for site managers to adjust resources to ensure smooth construction flows.

- Identifying threshold values for workers' presence in workspace. Another important objective of lean thinking is to eliminate waste onsite. Waste refers to the activities that consume resources but cannot add value for the process. Zhao et al. (2019) have proposed the concept of threshold value for uninterrupted presence onsite where they reasoned that a worker needs to stay at a work location for a certain period so that it can be counted as uninterrupted time, which is correlated with value-adding time. Because heat maps are generated on a basis of the density of onsite presence for identification of workspace, the actual threshold minute should be calculated according to the heat map of that worker in question where his or her workspace is detected. Therefore, the density of the presence of that worker in question should be an indicator of actual threshold value of his or her uninterrupted presence onsite rather than predefined hypothesis of " 1,5 and 10 minutes" in previous research (Zhao et al, 2019). For instance, heat maps can 
outline the border between workers' focused workspace (deeper color) and other random places (lighter color) they have been. Thus, the density of deeper-colored area forms a presence threshold value within workers' focused workspace. Compared to predefined presence threshold values in previous study, this method to determine the threshold minute of workers' presence onsite has positive contribution to evaluating workers' uninterrupted presence which is correlated with value-adding time.

- Forecasting potential workspace congestion. Akinci et al. (2002) have established an automated mechanism where they aimed to carry out projectspecific spaces required by site activities, which is presented by four dimensions: quantitative descriptions of the length in $\mathrm{x}, \mathrm{y}$ and $\mathrm{z}$, and the required times for each space. Heat maps represent the workers' real presence onsite that includes those dimensions to outline an estimation of workspace at a specific work location and timestamp. Time-space conflicts in construction happen frequently where task activities have a temporal aspect (Akinci et al, 2002). Heat maps generated by real-time tracking system can be used for predicting interfering degree among different tasks during the same time duration because the system supports multiresource tracking and generation of heatmaps based on overall movement density of given numbers of workers. For instance, if a certain area is detected with very high density of presence onsite at the same time from several task crews, the heat maps could raise alarms in advance for site managers to notice the potential work congestion in this area.

\section{DISCUSSION}

\section{IMPLICATION}

Our findings of the heat map application generated by real-time tracking system indicate that it can provide both dimensional and temporal data through an automated and illustrative process in the construction environment that has often been characterized as chaotic. Onsite temporal data such as time spent of workers and uninterrupted presence index connects with value-adding activities. For instance, Zhao et al. (2019) has argued that presence is necessary but not a sufficient condition for value-adding time, nevertheless it is reasonable to assume that value-adding time increases as share of worker's presence in work locations increases. Furthermore, onsite dimensional data such as working space area links with resource management such as labor, equipment and material. Heat maps that contains spatiotemporal data of both can generate a more straightforward space-loaded production model where value-adding activities can be potentially detected based on movement density of workers, and onsite resource handling can be optimized.

Single detected point with coordinate onsite is subject to system inaccuracy and variation, but heat maps can build a fuzzy outline to estimate workspace based on the density of onsite uninterrupted presence. The heat-map color change figure can help site managers to quickly identify the intensive working activities taken place at a certain time and place, and a dynamic heat map figure can be used to forecast potential workspace congestion in real practice. 


\section{LIMITATION}

The limitation of the method in the paper comes from the system inaccuracy for the detection of coordinates. The applied tracking system generates a researcher's coordinates for an average deviation of 10.69 meters in the best case of validation process. Therefore, single point is lacking strong evidence to be accurately detected onsite. This could hinder the generation of heat maps especially for identifying the border of estimate workspace. However, this paper demonstrated a prototype proof-of-concept implementation and it is possible to implement a commercial solution with much better accuracy (e.g. 1-3 m from a WIFI-based commercial positioning solutions (Woo et al. 2011)), but investments required for the system are increasing depending on the level of detail. We think the results based on this accuracy can provide a pilot dataset to analyze the proposed use cases in the paper for lean purposes.

Furthermore, though heat maps can estimate general workspace and complement other lean approaches in construction such as Takt area detection, what has actually happened onsite remains unknown only based on heat maps. Therefore, a more advanced positioning system featured with more accurate coordinate detection should be applied in future research, with help of other sophisticated validation methods such as vision-based technologies.

\section{CONCLUSION}

The paper has illustrated a case study where a BLE based real-time tracking system is implemented to capture workers' coordinate data in a construction site. The research is followed by onsite system implementation, coordinate detection, validation process, heat map generation and use proposals of heat maps. Though we find that single point of coordinate in this tracking method is subject to inaccuracy, the heat map generation based on density of workers' onsite presence can outline an estimate of workspace with fuzzy borders. Based on this, we propose the use cases from heat map application in construction that can detect takt areas in takt time planning, estimate threshold value of uninterrupted presence of workers, and forecast potential workspace congestion. The heat map approach applied in construction provides an innovative alternative to estimate workspace onsite with spatiotemporal data obtained by real-time tracking system. In future research, the result from this method is recommended to be validated by visionbased technologies for better model establishment and refinement.

\section{ACKNOWLEDGMENTS}

This work was supported by DiCtion (Digitalizing Construction Workflows) project funded by Business Finland and a consortium of companies.

\section{REFERENCES}

Alarcon, L.F. (ed.). (1997). Lean Construction. A.A. Balkema, Rotterdam, The Netherlands.

Akhavian, R., and Behzadan, A. H. (2016). "Smartphone-based construction workers' activity recognition and classification." Automation in Construction, 71, 198-209.

Akinci, B., Fischer, M., and Kunz, J. (2002). "Automated generation of work spaces required by construction activities." J. Constr. Engin. and Manage., 128(4), 306-315.

Akinci, B., Fischen, M., Levitt, R., and Carlson, R. (2002). "Formalization and automation of time-space conflict analysis." J. Comp. in Civil Engrg., 16(2), 124-134. 
Arditi, D., and Mochtar, K. (2000). "Trends in productivity improvement in the US construction industry." Construction Management and Economics, 18(1), 15-27.

Badihi , B., Zhao, J., Zhuang, S., Seppänen, O., and Jäntti, R. (2019). "Intelligent Construction Site: On Low Cost Automated Indoor Localization Using Bluetooth Low Energy Beacons.” In: 2019 IEEE Conf. on Wireless Sensors (ICWiSe), Pulau Pinang, Malaysia, pp. 29-35.

Emdanat, S., Linnik, M., and Christian, D. (2016). "A framework for integrating Takt planning, last planner system and labor tracking." Proc. 24 th Ann. Conf. Int. Group for Lean Construction, Boston, Mass., USA, Vol. 2, pp. 53-62.

Frandson, A.G., Seppänen, O., and Tommelein, I.D. (2015). "Comparison between location based management and takt time planning." Proc $23^{\text {rd }}$ Ann. Conf. Int. Group for Lean Construction, Perth, Australia, pp. 28-31.

Golovina, O., Teizer, J., and Pradhananga, N. (2016). "Heat map generation for predictive safety planning: Preventing struck-by and near miss interactions between workers-onfoot and construction equipment." Automation in Construction, 71, 99-115.

Hopp, W.J. and Spearman, M.L. (2008). "Shop Floor Control." In: Hopp and Spearman, (ed.) Factory Physics. Long Grove: Waveland Press.

Seppänen, O., Ballard, G., and Pesonen, S. (2010). "The Combination of Last Planner System and Location-Based Management System." Lean Construction J., 6 (1) 43-54, https://www.leanconstruction.org/media/docs/lcj/2010/LCJ_10_014.pdf

Teizer, J., Caldas, C.H., and Haas, C.T. (2007). "Real-time three-dimensional occupancy grid modeling for the detection and tracking of construction resources." J. Constr. Engin. and Manage., 133(11), 880-888.

Thomas, H.R., Horman, M.J., De Souza, U.E.L., and Zavřski, I. (2002). "Reducing variability to improve performance as a lean construction principle." J. Constr. Engin. and Manage., 128(2), 144-154.

Woo, S., Jeong, S., Mok, E., Xia, L., Choi, C., Pyeon, M., and Heo, J. (2011). "Application of WiFi-based indoor positioning system for labor tracking at construction sites: A case study in Guangzhou MTR." Automation in Construction, 20 (1) 3-13, doi.org/10.1016/j.autcon.2010.07.009.

Zhao, J., Seppänen, O., Peltokorpi, A., Badihi , B., and Olivieri, H. (2019). "Real-time resource tracking for analyzing value-adding time in construction." Automation in Construction, 104:52-65, doi.org/10.1016/j.autcon.2019.04.003

Zhao, J., Olivieri, H., Seppänen, O., Peltokorpi, A., Badihi , B., and Lundström, P. (2018). "Data Analysis on Applying Real Time Tracking in Production Control of Construction." In: IEEE Int. Conf. on Industrial Engineering and Engineering Management (IEEM), pp. 573-577, doi.org/10.1109/IEEM.2017.8289956. 